\title{
Uso de âncoras verbais em sistema fuzzy para facilitar a criação de mapas estratégicos iniciais: um estudo de caso
}

\author{
Alternative title: Verbal anchor usage on fuzzy systems to help creating initial \\ strategy maps: case study
}

\author{
Marcelo Ladeira \\ Departamento de Ciência da Computção \\ Universidade de Brasília \\ mladeira@unb.br
}

\author{
Fernando de Albuquerque Linhares \\ Departamento de Ciência da Computação \\ Universidade de Brasília \\ falinhares@unb.br
}

\begin{abstract}
RESUMO
Mais de $50 \%$ das empresas no Brasil não atinge o segundo ano de vida por falta de uma estratégia adequada. A metodologia Balanced Scorecard(BSC) foi proposta para auxiliar empresas criar planejamento estratégico simples que possa ser explicado para todos os funcionários de uma empresa. O ponto principal desta metodologia é o mapa estratégico que apresenta uma série de objetivos estratégicos que a empresa precisa atingir para a atingir sua missão. Empresas de pequeno e médio porte têm dificuldade em conseguir criar as suas estratégias sem o auxílio de consultores que nem sempre estão ao alcance de seus orçamentos. O Mistral Solutions foi desenvolvido para auxiliar empresários a construir mapas estratégicos, segundo a metodologia BSC. Para facilitar a aquisição de informação numérica, o administrador pode utilizar uma expressão linguística (âncoras verbais) que representa valores em uma escala. O sistema é baseado em lógica fuzzy e as regras são instanciadas a partir das respostas dadas e indicam os objetivos estratégicos mais adequados, em função dessas respostas. Esse artigo apresenta três estudos de casos de uso do Mistral Solutions no Tribunal Regional do Trabalho da $10^{\text {a }}$ Região, em uma companhia de construção civil, e em um cartório de registro de imóveis. Os mapas estratégicos propostos foram validados empiricamente por uma especialista em BSC e pelos encarregados de gerenciarem o planejamento estratégico dessas instituições. Essas instituições foram escolhidas porque elas representam os setores público, privado, e de concessões públicas. Para cada uma dessas instituições o Mistral Solutions selecionou os oito objetivos estratégicos mais adequados a partir de um conjunto de quarenta e cinco objetivos, dois para cada uma das perspectivas clássicas do BSC. Na avaliação empírica, os melhores resultados foram obtidos aplicando o sistema a empresas do setor privado, sendo os oito objetivos estratégicos selecionados considerados adequados pelo responsável pelo planejamento estratégico da instituição.
\end{abstract}

Permission to make digital or hard copies of all or part of this work for personal or classroom use is granted without fee provided that copies are not made or distributed for profit or commercial advantage and that copies bear this notice and the full citation on the first page. To copy otherwise, to republish, to post on servers or to redistribute to lists, requires prior specific permission and/or a fee.

SBSI 2015, May 26th-29th, 2015, Goiânia, Goiás, Brazil

Copyright SBC 2015.

\section{Palavras-Chave}

Sistemas Fuzzy, Mapas Estratégicos, Balanced Scorecard

\begin{abstract}
More than $50 \%$ of the Brazilian companies do not survive to its second year due to the lack of a proper business strategy plan. The Balanced Scorecard(BSC) methodology was proposed to help companies create simple strategy plans that can be explained to all employees within all company levels. The core of this methodology is the strategy map that shows a collection of strategic objectives that a company needs to achieve to reach its mission. Small and medium companies find it difficult to create their own strategies without the help of a management consultant, which is not always affordable. We proposed the Mistral Solutions, a system that helps managers to build strategy maps according to the BSC methodology. To easy the numeric knowledge acquisition process, a manager can answer a question using a verbal anchor scale instead of a number. This system is based on fuzzy logic. Fuzzy rules are instantiated from the answers given and indicate the most adequate strategic objectives. This paper presents three study cases based on the use of the Mistral Solutions. The Regional Labor Court of the $10^{\mathrm{a}}$ Region, a company in the civil construction industry, and a Real Estate Registry Office are focused as case of study. The strategy maps proposed by Mistral Solutions were empirically validated by a BSC specialist and by managers in charge of strategic planning of these institutions. These institutions represent the public sector, the private sector, and a public concession. Mistral Solutions selected the eight more appropriate strategic objectives from a set of forty five, two for each classic BSC perspectives. In the empirical evaluation, the system performed better when applied to private sector institutions when all the eight strategic objectives were considered adequate by the manager in charge of strategic planning of this institution.
\end{abstract}

\section{Categories and Subject Descriptors}

J.1 [Administrative Data Processing]: Business

\section{General Terms}

Management, Economics, Experimentation 


\section{Keywords}

Fuzzy Systems, Strategy Maps, Balanced Scorecard

\section{INTRODUÇÃO}

Segundo Kaplan e Norton[7], em uma pesquisa realizada em 2008 com um universo de 143 empresas americanas, $47,4 \%$ delas não conseguem chegar ao quarto ano de vida, sendo que somente $5 \%$ dos funcionários de todas as empresas pesquisadas entendem a palavra estratégia. Somente $25 \%$ dos gerentes destas empresas, possuem incentivos (financeiros ou não) conectados a estratégia da empresa e $85 \%$ dos executivos gastam menos de uma hora por mês discutindo essa estratégia. Entre as empresas, 9 em 10 falham em executar as estratégias por elas mesmas traçadas, sendo que $60 \%$ destas não propõem orçamentos compatíveis com a estratégia. Esses autores relacionam a falta de sucesso da empresa às dificuldades com a estratégia. Como o cenário internacional sobre estratégia empresarial é crítico, pressupõese ser ainda pior no Brasil, embora não se tenha encontrado nenhuma pesquisa similar com dados de empresas nacionais. É muito comum, ao se abordar o empresariado brasileiro e perguntar sobre estratégia, ouvir: "não, nós tomamos ações e corrigimos o barco ao longo do caminho.".

Segundo o Serviço Brasileiro de Apoio as Pequenas e Médias Empresas (SEBRAE)[10], a taxa de mortalidade apresentada pelas empresas no Brasil em 2002 indicava que 49,4\% não atingia dois anos de existência, 56,4\%, não atingiram três anos de vida e 59,9\% não chegaram ao quarto ano. Este mesmo estudo, atualizado em 2012 [11] indica que empresas constituídas em 2007 apresentavam uma taxa de sobrevivência de 75,6\% até o segundo ano. Em matéria de 04/04/2012, O SEBRAE listou os seis maiores erros de quem vai a falência e entre eles indica a falta de planejamento e o fato de se copiar modelos existentes, ambos os fatores indicadores de baixo ou nenhum uso de estratégia. Este estudo abrangeu um universo de 735 empresas e cada empresário pode apresentar um ou mais motivos listados na Tabela 1.

Tabela 1: Razões para o Fechamento de Empresas.

\begin{tabular}{|c|c|}
\hline Principais Razões & $\%$ \\
\hline Falta de Capital de Giro & 45,8 \\
\hline Carga Tributária Elevada & 41,7 \\
\hline Concorrência Muito Forte & 33,3 \\
\hline Maus Pagadores & 20,8 \\
\hline Falta de Clientes & 12,5 \\
\hline Ponto/Local Inadequado & 12,5 \\
\hline Desconhecimento do Mercado & 8,3 \\
\hline Recessão Econômica do País & 8,3 \\
\hline Descumprimento do Contrato & 4,2 \\
\hline Falta de Crédito Bancário & 4,2 \\
\hline Problemas com a Fiscalização & 4,2 \\
\hline Motivos Pessoais & 4,1 \\
\hline
\end{tabular}

Pode-se afirmar, com grande chance de acerto, que o planejamento estratégico em empresas de pequeno e médio porte no Brasil é negligenciado ou não existe, e que tais empresários não possuem ferramentas ou métricas à sua disposição que permitam uma análise mais sensível da situação de sua empresa ou do mundo ao seu redor. O mercado não dispõe de consultores a preços acessíveis que possam ajuda estes empresários no processo de construção e manutenção de estratégias. Também é comum que eles não possuam formação em Administração que lhes facilite elaborar o planejamento estratégico das suas empresas. A disponibilidade de um software que sugira objetivos estratégicos mas não requeira um especialista para ser utilizado, pode auxiliar os empresários a estabelecerem estratégias úteis para o planejamento estratégico.

Todos estes problemas apontam para a possível carência de um software que ajude a empresários não só a manter estratégias, bem como os ajude a montar tais estratégias. Se este software estivesse disponível e fosse de fácil acesso, parte dos problemas acima apontados poderiam ser contornados.

Esse artigo apresenta estudos de casos realizados com o Mistral Solutions, uma ferramenta que auxilie empresários e suas equipes a criar estratégias de simples entendimento para os funcionários de empresas, facilitando assim a adoção desta estratégia pela empresa. Esse sistema, baseado em Lógica Fuzzy, interage com o empresário e suas equipes através de um questionário online, cujas respostas disparam regras fuzzy, resultando na sugestão de objetivos estratégicos para as perspectivas básicas da metodologia do BSC: financeira, clientes, processos internos, e aprendizado \& inovação. O uso de âncoras verbais [9] possibilita ao empresário utilizar uma escala de expressões linguísticas para responder perguntas que requerem informação numérica.

O processo usual de se montar um BSC envolve uma série de entrevistas entre um especialista em estratégias empresariais e o gestor da empresa e sua equipe. Após a condução destas entrevistas, o especialista prepara um rascunho de um mapa estratégico, apresenta ao empresário que faz comentários, discute o mapa com o especialista e, eventualmente, faz ajustes no mesmo, finalizando o trabalho após algumas reuniões. A criação de mapas estratégicos requer a consultoria de um especialista em BSC pois mapas estratégicos resumem um planejamento estratégico a uma página com no máximo 30 objetivos estratégicos. Um típico documento de planejamento estratégico, equivalente a um mapa estratégico, possui cerca de 50 páginas. Para realizar a tarefa de criar um mapa estratégico, o consultor deve ter uma habilidade ímpar de síntese e compreensão das reais prioridades da empresa. A contribuição desse artigo é apresentar evidências empíricas de que é possível a um micro ou pequeno empresário selecionar objetivos e construir um mapa estratégico inicial, utilizando o Mistral Solutions, sem o apoio de um consultor especializado.

Não foi localizada na pesquisa bibliográfica realizada, referência a um sistema implementado que apoie a construção de um mapa estratégico com base na metodologia do BSC. Os sistemas existentes mais conhecidos como o Executive Strategy Manage, o Stratec, o Quickscore, o BSC Designer, o SAP BI, o Oracle Hyperion, e o BSC Designer são utilizados para facilitar a visualização após a conclusão do mapa estratégico e não para guiar o empresário e sua equipe durante a fase de criação do mapa.

Esse artigo está estruturado da seguinte forma: a Seção 2 apresenta os conceitos básico da metodologia BSC, a Seção 3 exemplifica o funcionamento do Mistral Solutions, a Seção 4 descreve os estudos de casos realizados. Por fim, a Seção 5 apresenta as conclusões e aperfeiçoamentos que podem ser realizados.

\section{O BALANCED SCORECARD RESUMIDO}


Os conceitos de planejamento estratégico empresarial são objetos de estudo em Administração e em Economia. No campo do planejamento estratégico, ligados a área de estudos de estratégia aplicada a negócios, diversos métodos são utilizados como as análises SWOT[2] e Porter Five Forces[8]. No entanto, somente o BSC apresenta métricas e iniciativas conectadas às métricas, as quais são mensuradas ao longo de um período de tempo.

A metodologia do BSC consiste em traduzir estratégias em diagramas de fácil leitura, análise e visualização, permitindo assim sua ampla divulgação e adoção por todos os funcionários de uma empresa como norte a ser seguido na busca de um objetivo comum. Um mapa estratégico é um subproduto oriundo dessa metodologia, criada pelos Professores Kaplan e Norton da Universidade de Harvard [7][3][4][5][6].

O BSC e seus mapas estratégicos adotam os seguintes conceitos[5]:

- toda empresa precisa ter uma visão, missão e valores.

- uma empresa não deve ter uma estratégia complexa para se manter no mercado.

- a estratégia de uma empresa deve ser abordada em quatro perspectivas:

- Perspectiva Financeira - Com o atendimento ao cliente bem feito, como a empresa é capaz de dar o retorno financeiro desejado?

- Perspectiva dos Clientes - Com processos internos bem feitos, como a empresa é capaz de atender bem ao cliente?

- Perspectiva dos Processos Internos - Com os seus funcionários treinados e com novos produtos e serviços, como a empresa é capaz de gerir os seus processos internos?

- Perspectiva do Aprendizado e Inovação - Como a empresa cria novos produtos e serviços e como ela prepara e treina os seus funcionários?

- dentro de cada perspectiva podem haver um ou mais objetivos estratégicos - frases que indicam proposições que a empresa deseja alcançar. Por exemplo: "Reduzir custos operacionais na linha de produção".

- todo objetivo estratégico deverá ter uma ou mais métricas associadas a ele de forma que permita a sua medição e acompanhamento.

- cada métrica deverá ter uma meta e prazos para mudanças de metas.

- iniciativas deverão ser criadas para que as métricas possam convergir para as metas. Estas iniciativas devem ser acompanhadas pelos gestores da empresa.

A Figura 1, adaptada de [5], ilustra um mapa estratégico.

\section{MISTRAL SOLUTIONS}

No site www.mistralsolutions.com.br pode-se acessar o Mistral Solutions. Esse sistema, desenvolvido em uma pesquisa de Mestrado em Informática [1], é baseado no uso do raciocínio e da inferência fuzzy[13] para lidar com incerteza de origem linguística, e de âncoras verbais [9]para representar valores numéricos sobre os quais não se tem precisão. O raciocínio fuzzy é baseado na teoria dos conjuntos

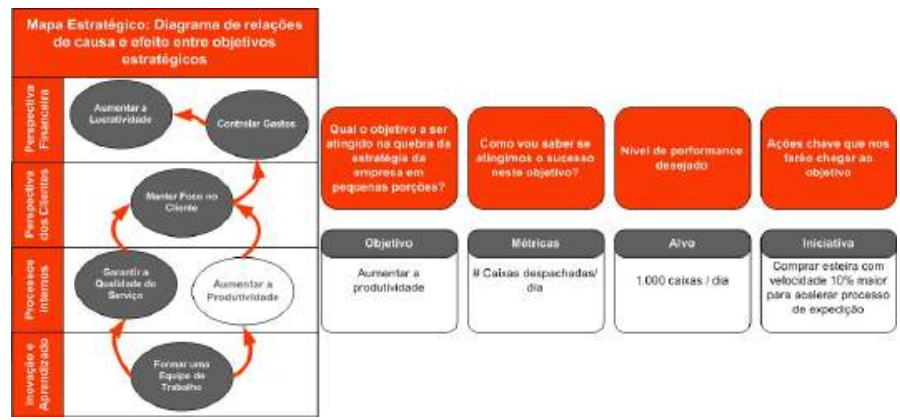

Figura 1: Mapa Estratégico

fuzzy de Zadeh[12] e está vinculado à representação do conhecimento por meio de regras do tipo $\mathrm{SE}<$ antecedente> ENTÃO <consequente>. O antecedente estabelece o contexto para o disparo dessa regra. A regra sendo disparada, resultará na execução das ações especificadas no seu consequente. Tanto o antecedente quanto o consequente de uma regra fuzzy podem conter variáveis fuzzy que podem ser vistas como conjuntos fuzzy com uma função de pertinência, ou seja conjuntos com fronteiras mal definidas. Um função de pertinência assume valores entre zero (o elemento não pertence ao conjunto) e um (o elemento pertence ao conjunto). O raciocínio fuzzy permite lidar com a incerteza da pertinência a um conjunto.

No início de um caso, o empresário, usuário do sistema responde a um questionário online, contendo 51 perguntas, sobre a situação da sua empresa. Este questionário permite a entrada de dados tanto na forma quantitativa quanto qualitativa, por meio do uso de âncoras verbais (Figura 2). É esperado que essas respostas não sejam muito precisas devido à possível falta de planejamento e de organização da empresa. As respostas dadas são utilizadas para instanciar os valores de 51 variáveis de entrada que fazem parte dos antecedentes das 126 regra fuzzy constantes na base de conhecimento do Mistral Solutions.

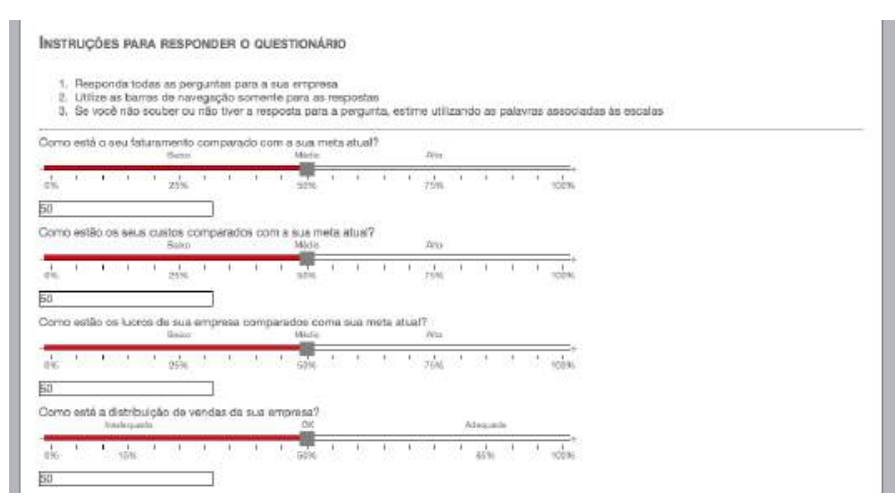

Figura 2: Entrada de Dados no Mistral Solutions

O sistema seleciona as variáveis de entrada, propaga as regras fuzzy compatíveis com as respostas do empresário, desfuzzifica o resultado aplicando o método do centro de gravidade da área resultante das interseção das regras disparadas, e retorna um valor entre 0 e 100, associado à variável de saída, indicando sua adequação. Cada uma das 
variáveis de saída representa um objetivo estratégico. Os dois objetivos mais adequações, segundo o valor obtido na defuzzificação, para cada perspectiva BSC, são selecionados e propostos pelo sistema.

O questionário apresentado pelo sistema foi elaborado com base na experiência de um dos autores desse artigo, o qual atua há onze anos como consultor em planejamento estratégico, construção de mapas estratégicos e implantação da BSC em empresas.

Como exemplo ilustrativo da aplicação da Lógica Fuzzy, retirado da base de conhecimento do sistema, tem-se a determinação da necessidade de se indicar melhorar ou não o sistema de controle de custos da empresa (variável Melhorar Sistema de Custos), com base na informação percentual (em relação à meta estabelecida) do atual nível de gastos da empresa (variável Custos) e a empresa ter ou não um sistema de controle de custos (variável Sistema de Custos). O valor da variável Melhorar Sistema de Custos é determinado por regras fuzzy, podendo ser Baixo, Médio ou Alto. A incerteza linguística associada a esses valores Baixo, Médio e Alto é modelada pela função de pertinências apresentada na Figura 3 .

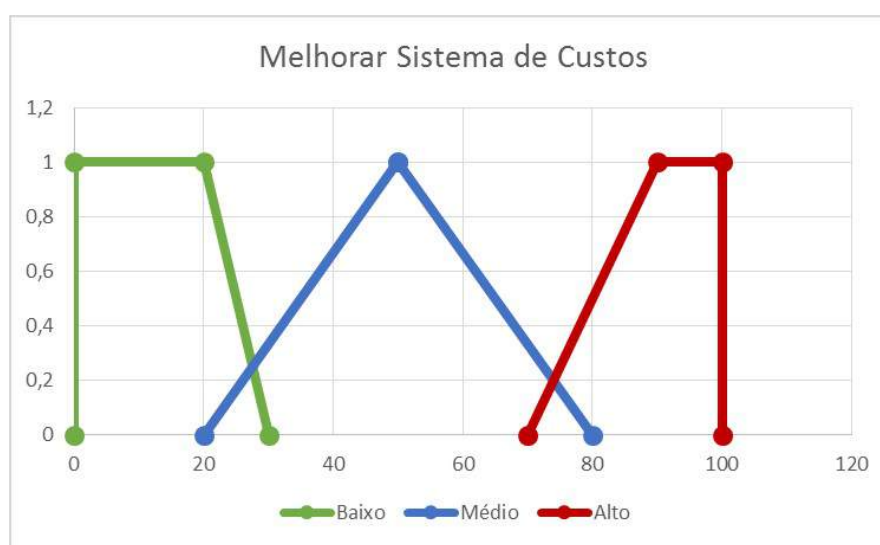

Figura 3: Função de pertinência para Melhorar Sistema de Custos

O empresário pode informar uma valor percentual para a variável Custos ou, se não o souber, poderá entrar com uma das âncoras verbais Baixo, Médio ou Alto. A incerteza linguística associada a esses valores está representada pela função de pertinência ilustrada na Figura 4.

Para a variável Sistema de Custos, o empresário poderá responder utilizando uma escala numérica percentual onde zero corresponde a não ter e cem corresponde a empresa ter um sistema de custos. Por outro lado, poderá também informar um valor intermediário indicando que tem algum controle sobre os custos. Se optar por utilizar as âncoras verbais SIM ou Não, a incerteza de origem linguística associada será modelada pela função de pertinência apresentada na Figura 5.

Para exemplificar o funcionamento do Mistral Solutions considere que o empresário entrou com Custos $=25 \%$ e Sistema de Custos $=100 \%$. Entrando-se com esses valores, respectivamente, nas funções de pertinência apresentadas nas Figuras 4 e 5, obtêm-se os graus de pertinências apresentados nas Tabelas 2 e 3.

Esses graus de pertinência são utilizados para disparar as seguintes regras fuzzy:

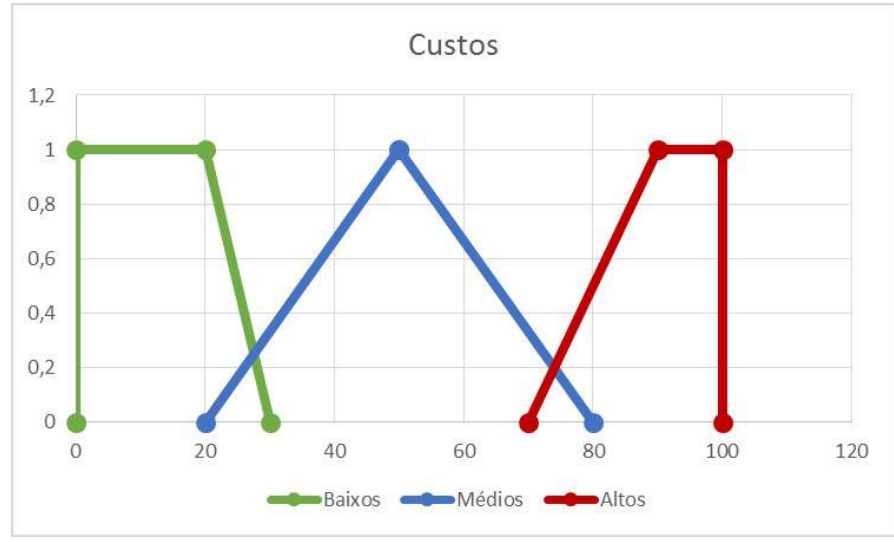

Figura 4: Função de pertinência para Custos

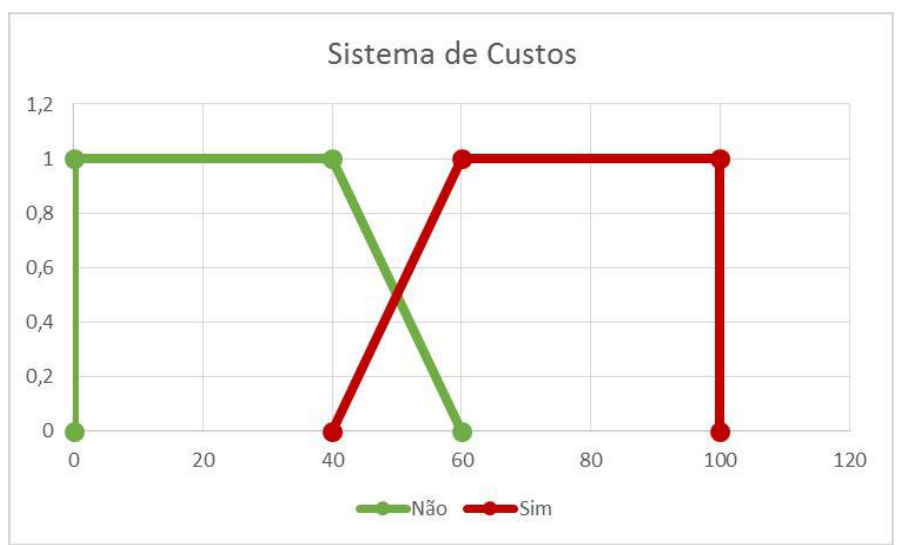

Figura 5: Função de pertinência para Sistema de Custos

1. IF (Sistema de Custos IS não) AND (Custo IS alto OR Custo IS médio) THEN Melhorar Sistema de Custos IS alto

2. IF (Sistema de Custos IS não) AND (Custo IS baixo) THEN Melhorar Sistema de Custos IS médio

3. IF (Sistema de Custos IS sim) THEN Melhorar Sistema de Custos IS Baixo

resultando nos graus de pertinência para Melhorar Sistema de Custo, apresentados na Tabela 4. A defuzzificação é feita pelo método do Centro de Gravidade aplicado à área definida por esses graus de pertinência (Figura 6) sobre a função de pertinência da variável Melhorar Sistema de Custos.

Valor(aproximado): Necessidade de Melhorar Sistema de Custos $=0,17 * 100+(0,5-0,17) * \frac{(46,5+30)}{2}+(1-0,17) *$ $\frac{(27,5+20)}{2}=49 \%$

\subsection{Arquitetura do Mistral Solutions}

O sistema foi construido utilizando orientação a objetos e o modelo de três camadas:

- camada de apresentação - Esta camada interage diretamente com o usuário. Apresenta os questionários construidos com as respectivas âncoras verbais. Os objetivos estraégicos sugeridos são apresentados em um relatório nesta camada. 
Tabela 2: Graus de Pertinência para Custos

\begin{tabular}{|c|c|c|c|c|}
\hline Custos & Valor Real & Baixo & Médio & Alto \\
\hline & $25 \%$ & 0,50 & 0,17 & 0,00 \\
\hline
\end{tabular}

Tabela 3: Graus de Pertinência para Sistema de Custos

\begin{tabular}{|c|c|c|c|}
\hline Sistema de Custos & Valor Real & Não & Sim \\
\hline & $100 \%$ & 0,00 & 1,00 \\
\hline
\end{tabular}

- camada de negócios - Constituida pela biblioteca que implementa o raciocínio fuzzy para determinação dos objetivos estratégicos.

- camada de dados - Constituida pelas regras fuzzy, âncoras verbais e respostas dadas pelo usuário armazenadas em uma base de dados MySQL.

O sistema foi construido utilizando os seguintes frameworks:

- Visual Studio Professional 2010 com linguagem C\#

- Biblioteca Fuzzy DotFuzzy em linguagem C\# ${ }^{1}$

- Dojo Javascript toolkit, para a criação das escalas com âncoras verbais

- Base de dados MySQL

- Hospedagem em servidor Windows Server 2012 dedicado

O resultado é um portal ASPX.NET hospedado no site www.mistralsolutions.com.br que permite o cadastro de usuários, coleta de dados, visualização e envio de relatórios dos dados entrados e resultados obtidos.

\section{ESTUDOS DE CASOS}

Após a proposição da base de conhecimentos, o acesso ao Mistral Solutions foi disponibilizado, via Internet, para empresários que responderam ao questionário e geraram objetivos estratégicos. Para cada uma das instituições que preencheu o questionário, o Mistral Solutions, com base nas respostas dadas, selecionou os oito objetivos estratégicos mais adequados, a partir de um conjunto de quarenta e cinco objetivos, sendo apresentados dois objetivos estratégicos para cada uma das perspectivas do BSC. As respostas dadas aos questionários e os objetivos estratégicos propostos pelo sistema foram analisados empiricamente por um especialista, consultor em planejamento estratégico segundo a metodologia do BSC. Esse especialista analisou a adequação desses objetivos em função das respostas apresentadas pelos empresários e suas equipes, e sugeriu alterações na base de conhecimento que foram implantadas.

Com a base de conhecimentos revista, para avaliar a aplicabilidade do Mistral Solutions a diversos tipos de instituições, três estudos de casos foram criados, abrangendo os setores público, privado e concessionário público. Em todos esses casos foi solicitado o preenchimento do questionário e a análise adequação dos objetivos estratégicos propostos pelo sistema. As instituições abrangidas nesses estudos de caso foram:

${ }^{1}$ https://github.com/MicheleBertoli/DotFuzzy
Tabela 4: Graus de Pertinência

\begin{tabular}{|c|c|}
\hline Melhorar Sistema de Custos & Valor \\
\hline Baixo & 1,00 \\
\hline Média & 0,50 \\
\hline Alto & 0,17 \\
\hline
\end{tabular}

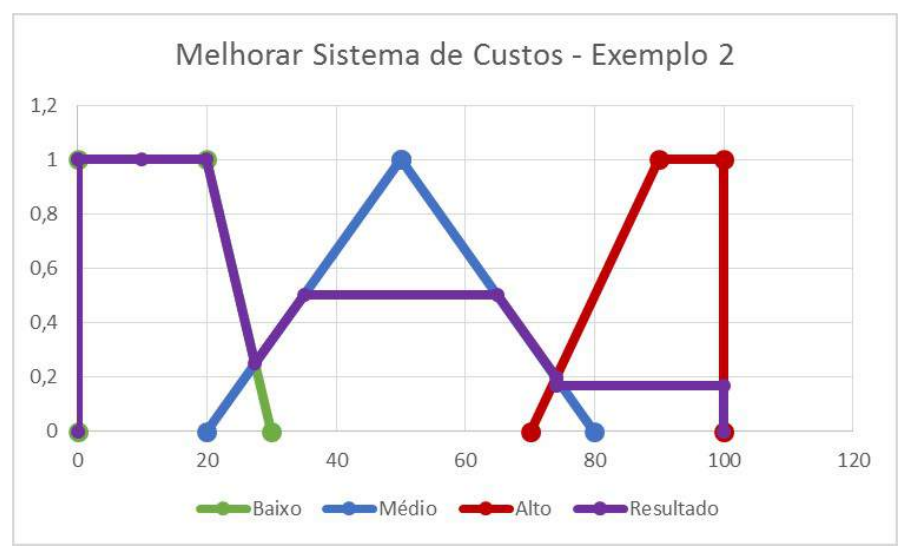

Figura 6: Desfuzzificação por centro de gravidade

- TRT da $10^{a}$ Região

- Empresa do ramo da construção civil

- Cartório de registro de imóveis

O questionário e as respostas dessas instituições estão apresentadas na Tabela 5. As âncoras verbais foram transformadas em percentagens, segundo a Tabela ??.

\subsection{TRT da $10^{a}$ Região}

O Tribunal Regional do Trabalho da $10^{(} a$ ) é um órgão público que possui planejamento estratégico há anos. A motivação desse estudo de caso foi verificar se os objetivos estratégicos, propostos pelo Mistral Solutions, são coerentes com os previstos no planejamento estratégico dessa instituição. Esses objetivos, listados a seguir, exceto o último, foram considerados válidos, correspondendo a uma taxa de sucesso de $87,5 \%$. Os valores entre parenteses, resultantes da defuzzificação, representam o nível de adequação associado ao objetivo estratégico. Como o questionário foi elaborado para empresas, algumas perguntas envolvendo "clientes", "vendas"ou "produtos"não se aplicam diretamente ao TRT e foram contextualizados, pelo respondente, para esse domínio. Assim, o objetivo estratégico "Desenvolver novo canal de vendas"é entendido como utilizar novos meios para ofertar os serviços prestados pelo TRT à população. O objetivo "Criar novos pontos de vendas"é entendido como ampliar o número de unidades (locais onde se ofertam serviços) do TRT. O objetivo estratégico "Fazer análise tributária"não foi considerado adequado e compatível com as atribuições do TRT. Os níveis de adequação associados aos seis primeiros objetivos estratégicos propostos, da ordem de valor de $50 \%$, indicam adequação mediana para o domínio público.

- Perspectiva do Aprendizado e Inovação

- Montar pipeline de inovações (49,90\%) 
- Criar programa de bonificação $(50,00 \%)$

- Perspectiva dos Clientes

- Aumentar a satisfação do cliente (50,00\%)

- Desenvolver novo canal de vendas $(50,00 \%)$

- Perspectiva dos Processos Internos

- Implementar sistema de controle orçamentário (50,00\%)

- Melhorar processos internos (50,00\%)

- Perspectiva Financeira

- Criar novos pontos de vendas $(89,17 \%)$

- Fazer análise tributária $(89,17 \%)$

\subsection{Empresa de Venda de Produtos para a Cons- trução Civil}

A empresa escolhida para esse estudo de caso possui 15 anos de atuação no mercado varejista. Embora a empresa não possua uma planejamento estratégico formal, esse empresário é experiente e executa um procedimento empírico e informal de planejamento estratégico. As respostas dadas por um empresário, sócio proprietário dessa empresa, ao questionário do Mistral Solutions estão apresentadas na Tabela 5. Os objetivos estratégicos propostos, e suas adequações, estão apresentados a seguir:

- Perspectiva do Aprendizado e Inovação

- Medir satisfação dos funcionários (89,17\%)

- Criar programa de treinamentos $(50,00 \%)$

- Perspectiva dos Clientes

- Medir satisfação do cliente $(89,17 \%)$

- Aumentar satisfação do cliente (64,40\%)

- Perspectiva dos Processos Internos

- Automatizar processos chave da empresa(89,17\%)

- Analisar políticas internas $(64,88 \%)$

- Perspectiva Financeira

- Criar novos pontos de vendas $(89,17 \%)$

- Reduzir os custos $(89,17 \%)$

Todos esses objetivos foram considerados adequados pelo empresário, constituindo uma taxa de sucesso de $100 \%$ para esse caso de estudo. Os valores associados a esses objetivos estratégicos são bem mais elevados do que os obtidos no caso de estudo TRT, indicando maior adequabilidade do uso do Mistral Solutions em empresas privadas.

\subsection{Cartório}

O Cartório de Registro de Imóveis selecionado realiza planejamento estratégico há alguns anos. O objetivo desse estudo de caso foi obter evidência empírica sobre a performance do Mistral Solutions em uma instituição com concessão pública, caracterizando um setor diferente dos setores público e do setor privado. Uma funcionária, responsável pelo planejamento estratégico do Cartório, rodou o sistema e validou os objetivos estratégicos propostos, comparando com os objetivos constantes do planejamento estratégico existente. Esses objetivos, e os níveis de adequação associados, resultantes do processo de desfuzzificação, estão apresentados a seguir. As respostas dadas pela funcionária às questões constantes do questionário estão apresentadas na Tabela 5.

- Perspectiva do Aprendizado e Inovação

- Criar programa de bonificação $(50,00 \%)$

- Melhorar comunicação interna (49,90\%)

- Perspectiva dos Clientes

- Desenvolver novo canal de vendas $(50,00 \%)$

- Fazer a voz do consumidor para marcas $(50,00 \%)$

- Perspectiva dos Processos Internos

- Mapear processos chave da empresa $(89,17 \%)$

- Melhorar processos internos $(67,16 \%)$

- Perspectiva Financeira

- Fazer análise tributária $(89,17 \%)$

- Reduzir os custos $(89,17 \%)$

Todos os objetivos propostos, exceto o primeiro, "Criar programa de bonificação", foram avaliados como adequados pela responsável pelo planejamento estratégico do Cartório, correspondendo a uma taxa de sucesso de $87,5 \%$. O objetivo estratégico "Fazer a voz do consumidor para marcas"constitui um jargão nessa área e significa entender o que o consumidor quer dos produtos e serviços que o Cartório pode oferecer. Para quatro dos objetivos propostos, os níveis de adequação associados são de $50 \%$, indicando uma performance mediana para esse setor de concessão pública, mas superior à performance apresentada no caso de estudo do TRT, um órgão público.

\section{CONCLUSÕES}

Foram apresentados três estudos de casos envolvendo uma instituição de cada um dos seguintes setores: público (tribunal TRT), privado (empresa de venda de produtos para a construção civil) e concessionário público (cartório de registro de imóveis). O TRT e o Cartório são instituições que realizam planejamento estratégico. A empresa não possui nenhum planejamento estratégico formal. Cada responsável pela realização do planejamento estratégico em cada uma dessas instituições foi entrevistado no sentido de que avaliasse se os objetivos estratégicos iniciais sugeridos estavam válidos para o momento atual da instituição ou para o futuro próximo. No geral, 7 em 8 objetivos sugeridos foram aceitos como válidos, levando o sistema a uma taxa mínima de acerto de $87,5 \%$. O melhor desempenho foi obtido para 
a empresa onde todos os objetivos estratégicos propostos foram considerados válidos.

Esses estudos de casos forneceram evidência empírica de que o Mistral Solutions constitui uma ferramenta útil para apoiar administradores a realizarem planejamento estratégico em suas instituições, seguindo a metodologia BSC, utilizando os objetivos estratégicos propostos pelo sistema como um ponto de partida para a elaboração de um mapa estratégico.

Não se encontrou na literatura analisada a indicação da existência de um sistema que auxiliasse o administrador a propor objetivos estratégicos. Todos os trabalhos encontrados partem do pressuposto da existência desses objetivos estratégicos.

\subsection{Trabalho futuro}

Para um melhor desenvolvimento do sistema, torna-se necessário:

- manter a divulgação do sistema em redes sociais e portais para aumentar o número de casos coletados e obtenção de feedbacks do uso do sistema que possam ser utilizados para a evolução da base de conhecimento;

- incluir a sugestão de métricas ao usuário associadas aos objetivos estratégicos;

- criar condições para que o Mistral Solutions seja uma solução completa de BSC;

- correlacionar medições de métricas com grupos de CNAE para estabelecer padrões por indústrias;

- oferecer o uso sem custos do sistema para base de clientes e potenciais clientes das empresas de que o mestrando é sócio, oferecendo serviços pró-bono limitados de consultoria para customizar as estratégias geradas pelo sistema;

- Pesquisar a comparação de mapas estratégicos iniciais gerados pelo sistema através da metodologia Fuzzy AHP. são:

Os passos previstos para a evolução do Mistral Solutions

- Validar e refinar a base de conhecimentos com mais especialistas

- Aumentar o número de estudos de casos

- Correlacionar medições de métricas, objetivos estratégicos sugeridos e CNAE de empresas para estabelecer benchmarketings de mercado com objetivos estratégicos mais adequados para grupos específicos de CNAE

- Evoluir sistema na implementação da metodologia completa dos 10 passos para atrair mais usuários

- Pesquisar a comparação de mapas estratégicos gerados pela metodologia do Fuzzy AHP.

\section{REFERÊNCIAS}

[1] F. de Albuquerque Linhares. Utilização de Âncoras Verbais e Raciocinio Fuzzy na Construção de Mapas Estratégicos da Metodologia Balanced Scorecard. M.Sc., Universidade de Brasília, Brasilia, Brazil, 2015.

[2] A. Humphrey. Swot analysis for management consulting. Stanford Research Institute (SRI International) Alumni Newsletter, pages 7-8, December 2005.

[3] R. S. Kaplan and D. P. Norton. The Balanced Scorecard: Translating Strategy into Action. Harvard Business Review Press, 1996.

[4] R. S. Kaplan and D. P. Norton. The Strategy-Focused Organization: How Balanced Scorecard Companies Thrive in the New Business Environment. Harvard Business Review Press, 2001.

[5] R. S. Kaplan and D. P. Norton. Strategy Maps: Converting Intangible Assets into Tangible Outcomes. Harvard Business Review Press, 2004.

[6] R. S. Kaplan and D. P. Norton. Alignment: Using the Balanced Scorecard to Create Corporate Synergies. Harvard Business Review Press, 2006.

[7] R. S. Kaplan and D. P. Norton. The Execution Premium: Linking Strategy to Operations for Competitive Advantage. Harvard Business Review Press, Boston, Massachusetts, 2008.

[8] M. E. Porter. The five competitive forces that shape strategy. Harvard Business Review, 2008(1):1-10, January 2008.

[9] S. Renooij and C. Witteman. Talking probabilities: communicating probabilistic information with words and numbers. International Journal of Approximate Reasoning, 22(3):169-194, 1999.

[10] SEBRAE. Fatores Condicionantes e Taxa de Mortalidade de Empresas no Brasil. SEBRAE, 2005.

[11] SEBRAE. Sobrevivência das empresas no Brasil. SEBRAE, 2013

[12] L. A. Zadeh. Fuzzy sets. Information and Control, 8(3):338-353, 1965.

[13] L. A. Zadeh. Fuzzy sets as a basis for a theory of possibility. Fuzzy Sets and Systems, 1(1):3-28, 1978.

\section{APÊNDICE}


Tabela 5: Perguntas e Respostas das Instituições

\begin{tabular}{|c|c|c|c|}
\hline Pergunta & TRT & Empresa & Cartório \\
\hline Como está o seu faturamento comparado com a sua meta atual? & 50 & 40 & 65 \\
\hline Como estão os seus custos comparados com a sua meta atual? & 50 & 100 & 50 \\
\hline Como estão os lucros de sua empresa comparados coma sua meta atual? & 50 & 100 & 35 \\
\hline Como está a distribuição de vendas da sua empresa? & 50 & 30 & 85 \\
\hline Como estão os seus impostos pagos comparados com o previsto? & 50 & 75 & 40 \\
\hline Sua empresa possui sistema de controle de custos? & 0 & 100 & 5 \\
\hline Qual é sua taxa de retorno sobre ativos (ROA) & 50 & 50 & 75 \\
\hline Como está sua venda média por cliente comparada com a sua meta? & 50 & 80 & 65 \\
\hline Sua empresa possui área de compras definida? & 100 & 100 & 100 \\
\hline Sua empresa possui programa de bonificação & 0 & 100 & 0 \\
\hline Sua empresa possui produtos de baixa margem e alto volume de vendas? & 50 & 100 & 100 \\
\hline Sua empresa concentra vendas em um canal ou cliente específico? & 50 & 100 & 100 \\
\hline Sua empresa controla as métricas que mede? & 100 & 100 & 100 \\
\hline Como está o seu custo de logística comparado com a meta atual? & 25 & 100 & 25 \\
\hline Você sabe dados da sua competição? & 100 & 0 & 75 \\
\hline Sua empresa tem descrição de cargos? & 100 & 75 & 100 \\
\hline Existe desperdício na sua empresa? & 100 & 70 & 50 \\
\hline Você documenta como as vendas são feitas? & 50 & 100 & 100 \\
\hline Existe algum dreno de custos na empresa? & 100 & 0 & 50 \\
\hline Qual o nível médio de educação dos funcionários? & 100 & 75 & 75 \\
\hline Faltam funcionários para executar funções na empresa? & 100 & 70 & 25 \\
\hline Qual o faturamento médio por cliente comparado com a meta atual? & 50 & 50 & 65 \\
\hline Todos na empresa sabem o que fazer? & 70 & 90 & 30 \\
\hline Sua empresa faz a voz do consumidor para produtos? & 100 & 20 & 0 \\
\hline Há distinção de gastos pessoais dos sócios dos gastos da empresa? & 100 & 100 & 100 \\
\hline Sua empresa tem métricas definidas? & 100 & 75 & 100 \\
\hline Sua empresa mede a satisfação dos clientes? & 100 & 15 & 100 \\
\hline Sua empresa mede a satisfação dos funcionários? & 100 & 15 & 100 \\
\hline Existe um plano definido para aumentar vendas? & 50 & 85 & 75 \\
\hline Quantos níveis hierárquicos existem na sua empresa? & 100 & 85 & 75 \\
\hline Você sabe quais são as oportunidades e ameaças para a sua empresa? & 100 & 85 & 50 \\
\hline Sua empresa possui um pipeline de inovações & 10 & 50 & 50 \\
\hline Sua empresa tem plano de carreiras? & 100 & 60 & 80 \\
\hline Sua empresa tem planejamento estratégico definido? & 100 & 60 & 100 \\
\hline Sua empresa possui plano de negócios definido? & 50 & 60 & 0 \\
\hline Qual é o porte da sua empresa? & 100 & 50 & 80 \\
\hline Qual é a posição no mercado da sua empresa? & 100 & 10 & 0 \\
\hline Sua empresa possui processos mapeados? & 15 & 75 & 10 \\
\hline Sua empresa vende primariamente produtos ou serviços? & 15 & 10 & 100 \\
\hline Sua empresa possui programa de estudos? & 100 & 20 & 50 \\
\hline Sua empresa tem programa de treinamento? & 100 & 35 & 100 \\
\hline Qual é a sua quantidade de clientes comparada com a sua meta? & 50 & 40 & 75 \\
\hline Qual é a sua quantidade de funcionários? & 100 & 50 & 100 \\
\hline Qual é a quantidade de produtos/serviços fornecidos pela sua empresa? & 50 & 100 & 0 \\
\hline Qual é o retorno de investimento da empresa comparado com a meta? & 50 & 100 & 100 \\
\hline Qual é o nível de satisfação dos seus clientes? & 30 & 75 & 95 \\
\hline Sua empresa possui produtos de alta margem e baixo volume de vendas? & 50 & 40 & 100 \\
\hline Você sabe as forças e fraquezas da sua empresa? & 100 & 75 & 50 \\
\hline O trabalho da sua empresa está documentado? & 75 & 75 & 25 \\
\hline Sua empresa possui valores definidos? & 100 & 75 & 100 \\
\hline Qual o valor de faturamento médio por produto comparado com a meta? & 50 & 50 & 60 \\
\hline Sua empresa executa vendas? & 0 & 100 & 100 \\
\hline Sua empresa tem um plano de comunicações? & 100 & 0 & 50 \\
\hline Sua empresa tem área de RH constituída? & 100 & 0 & 50 \\
\hline Qual é o nível de qualidade dos processos executados? & 50 & 50 & 65 \\
\hline Sua empresa tem bonificação atrelada à redução de custos na empresa? & 0 & 65 & 100 \\
\hline Qual é o nível de integração das equipes da empresa? & 25 & 25 & 10 \\
\hline Existem problemas disciplinares na sua empresa? & 10 & 100 & 80 \\
\hline
\end{tabular}

